\title{
Incidence of Secondary Microorganism Infections Following Utilization of Tocilizumab for the Treatment of COVID-19 - A Matched Retrospective Cohort Study
}

\author{
Joanna L. Moore*1, Stephanie J. Stroever, Patricia E. Rondain, Robyn N. Scatena \\ ${ }^{1}$ Department of Medicine, Nuvance Health, University of Vermont School of Medicine, Norwalk, Connecticut, USA \\ ${ }^{2}$ Department of Innovation and Research, Nuvance Health, Danbury, Connecticut
}

*Corresponding author: Dr. Joanna L. Moore, 34 Maple Street, Norwalk, Connecticut 06850, USA. E-mail: joanna.moore@ nuvancehealth.org

Citation: Moore JL, Stroever SJ, Rondain PE, Scatena RN. Incidence of secondary microorganism infections following utilization of tocilizumab for the treatment of COVID-19 - A matched retrospective cohort study published in Med Science Journals.

Received Date: 16 July 2021 Accepted in Revised Form: 28 July 2021 Published Date: 16 August 2021

\begin{abstract}
Introduction: immunological disorder agent's area unit theorized to focus on the protein storm syndrome in COVID-19. However, the downstream effects concerning susceptibilities to secondary infection risk stay unknown. This study seeks to work out risk variations for secondary infections among COVID-19 patients World Health Organization did and failed to receive tocilizumab. Methods: we have a tendency to conducted a matched retrospective cohort study from 2 giants, acute care hospitals in Western Connecticut from March 1 to May 31, 2020. we have a tendency to collected variables exploitation manual case history abstraction. the first exposure variable was any dose of tocilizumab. the first outcome was any healthcare-associated microorganism or mycosis as outlined by the National Care Safety Network. we have a tendency to performed a Kaplan-Meier analysis to assess the crude distinction within the additive likelihood of healthcare-associated infection (HAI) across exposure teams. we have a tendency to conjointly performed a multivariable Cox multivariate analysis to work out the hazard quantitative relation for HAI by exposure

group whereas dominant for potential confounders. Results: The Kaplan-Meier analysis incontestable no distinction within the additive likelihood of HAI across teams. The adjusted hazard of HAI for patients given tocilizumab was zero.85 times that of patients not given tocilizumab $(95 \%$ confidence interval $=$ zero.29, $2.52, P=0.780)$ once dominant for relevant confounders. Conclusions: Tocilizumab failed to increase the incidence of secondary infection among COVID-19 patients. Larger, irregular trials ought to valuate infection as a secondary outcome to validate this finding.
\end{abstract}

Keywords: Coronavirus, COVID-19, IL-6, SARS-CoV-2, secondary infection, tocilizumab

\section{Introduction}

Coronavirus-19 (COVID-19) is a novel infection that surged early in 2020 with restricted proof of effective treatment ways. The virus might end in well to delicate cases however is additionally capable of inflicting severe malady with high mortality in others. The late part of infection is characterised by pathological hyper-activation of the system and considerably elevated inflammatory cytokines like interleukin-6 (IL-6).[2-4] This protein unleash syndrome (CRS), or "cytokine storm," typically results in speedy clinical deterioration and death and needs swift management with medication to suppress the system. Tocilizumab (Genentech, point of entry, USA) could be a humanized recombinant antibody antibody receptor antagonist that has traditionally been accustomed treat autoimmune disorder. thought-about thought-about for the treatment of COVID-19, rheumatologists and alternative clinicians recommend it should be a great tool to combat CRS. However, most of the proof to support this approach is anecdotal or derived from little case studies. a probe of the U. S. National Library of drugs clinical trials written account known twenty-three protocols registered to check tocilizumab in COVID-19 patients. However, all either were within the preparation or accomplishment part or had been withdrawn utterly. Reports from clinical trials of tocilizumab in patients with autoimmune disorder documented associate degree exaggerated risk of significant infection in patients World Health Organization received the medication. The few studies that evaluated tocilizumab in COVID-19 patients documented secondary infections yet, although most didn't compare teams statistically. Given the mechanism of action and existing proof, it's a logical concern that tocilizumab might place patients at higher risk for secondary healthcare-associated infection (HAI). However, no studies so far have assessed the incidence of HAI in COVID patients that received tocilizumab compared to those that didn't. the first objective of this study was to see the distinction within the incidence of secondary healthcare-associated microorganism and fungous infections among patients with COVID-19 given exposure to 
tocilizumab. we tend to hypothesized that patients World Health Organization received tocilizumab throughout their patient treatment for COVID-19 had a bigger rate of HAI than those that didn't receive tocilizumab once dominant for relevant confounders. This study will give moderate proof to support clinical decision-making for COVID treatment whereas awaiting the results of clinical trials.

\section{Methods}

\section{Study design and sampling}

To meet our objective, we have a tendency to conducted a matched retrospective cohort study. we have a tendency to selected patients from 2 massive, acute care hospitals in western Connecticut heavily compact by the COVID-19 surge between March first, and will thirty first, 2020. we have a tendency to enclosed patients within the study if they were $>18$ years recent, admitted as inpatients throughout this timeframe, and beaked with the ICD-10 code for COVID-19 (U.07.1). we have a tendency to excluded patients if that they had a microorganism or zymosis gift at the time of admission or were taking medication medications. we have a tendency to more refined our sample by distinguishing all patients prescribed tocilizumab throughout their patient keep. we have a tendency to matched patients employing a $1: 1$ magnitude relation to develop a corresponding management cluster among patients World Health Organization failed to receive tocilizumab. we have a tendency to used individual matching by people (10-year increments), gender, and admission to the medical aid unit and excluded patients from the study if they failed to with success match with an effect. In total, there have been 128 total patients within the study with 64 in every cluster World Health Organization received and failed to receive tocilizumab, severally. we have a tendency to failed to follow patients on the far side time to event being either infection, discharge, or death.

\section{Measures}

We collected all variables victimisation manual anamnesis abstraction. the first outcome was any healthcare-associated microorganism or zymosis, as well as blood, tract, skin, soft tissue, organ-space, and respiratory disease. we have a tendency to used the National aid Safety Network (NHSN) definitions to identify infections each related to and freelance of invasive devices(i.e., central line, Foley tubing, ventilator). we have a tendency to documented patients as having the first outcome if they met all site-specific or device-associated criteria. Patients World Health Organization met some however not all criteria weren't documented as positive for the first outcome (i.e., positive excretory product culture with $<100,000 \mathrm{CFU} / \mathrm{ml}$ ). we have a tendency to additionally documented the infection event date for time-dependent analyses.

The primary exposure variable was any indefinite quantity of tocilizumab administered to a patient with COVID-19. we have a tendency to documented the date of initial administration for time-dependent analyses. we have a tendency to enclosed age as endless variable in our analysis since matches were performed in increments of 10years. we have a tendency to conjointly enclosed the central line, Foley tubing, and ventilator days as confounders, outlined because the range of days a patient had the invasive device from date of placement so far of ending. we have a tendency to conjointly enclosed enclosed that will contribute to infection like polygenic disease, active cancer, and body mass index (BMI) (continuous). Finally, we have a tendency to enclosed whether or not the patient received Plaquenil or had surgery throughout the relevant inmate keep.

\section{Statistical analyses}

We used Stata SE sixteen for all applied math analyses. we have a tendency to computed descriptive statistics to replicate the amount and proportion of patients for every categorical variable and mean with variance for continuous variables. we have a tendency to performed univariate analyses to assess exposure cluster variations with the Chi-square and freelance student's t-tests. there has been no missing information during this dataset, and also the alpha for all hypothesis testing was set a priori at zero.05. we have a tendency to enclosed tocilizumab as a time-varying variable all told analyses. Entry into the cohort began at admission for all patients to account for immortal time bias. The event date within the analysis corresponded with the infection event date outlined by NHSN. we have a tendency to failed to follow patients for infection postdischarge and expurgated patients at the date of discharge or death (for those that invalid while not experiencing the event). we have a tendency to performed a Kaplan-Meier analysis to assess the crude distinction within the additive likelihood of HAI across exposure teams and used the log-rank check to see the distinction in survival functions. we have a tendency to conjointly performed Cox multivariate analysis to see the hazard quantitative relation, ninety fifth confidence interval(CI), and P-value for HAI by exposure cluster. we have a tendency to enclosed age, central line, Foley tubing, and ventilator days, BMI, diabetes, cancer, administration of Plaquenil, and inmate surgery within the model to account for hypothesized unsupportive per the classical definition. every covariate has associate degree associate degree association with the end result and isn't within the causative pathway. Finally, we have a tendency to verified the idea for proportional hazards by examining Schoenfeld residuals. 


\section{Results}

\section{Sample characteristics}

We know sixty-seven patients UN agency met the inclusion criteria and received tocilizumab throughout their patient stays. 3 patients failed to match with any controls and were eliminated from the sample. Of note, 2 of 3 patients were between eighteen and twenty-one years previous. Given the low prevalence of COVID-19 during this age bracket throughout the surge, it's not stunning they didn't match. The remaining sixty-four patients matched with success, and that we reviewed 128 patient records for exclusion criteria. we tend to known eighteen patients with microorganism or flora infections gift on admission and removed them from the sample (Nunexposed $=13$; Nexposed $=5$ ). One patient transferred in from another facility and failed to have info obtainable concerning infections at the time of admission. at the moment exclusion, the ultimate sample size was 109 . The mean age of patients within the sample was fifty-six.3 years, and therefore the majority of the sample was male (73.4\%) and preponderantly preponderantly $(52.3 \%$ ) [Table 1]. the bulk of patients UN agency failed to determine as a race were Hispanic (77.1\%). there have been no exposure cluster variations in any of the covariates, although the mean length of follow-up time within the cohort was longer within the exposed cluster than the unexposed cluster (10.7 days vs. 7.9 days, severally). The median time from admission to tocilizumab administration was three days (range $=0-12$ ).

\section{Kaplan-Meier analysis}

There were nineteen HAIs known within the sample. The Kaplan-Meier survival curve was stratified by exposure cluster. we tend to found there wasn't a statistically vital distinction within the time to event across exposure teams (log-rank test: $\mathrm{P}=\mathrm{zero} .81)$.

\section{Cox regression}

We verified that the crude and full models met the belief of proportional hazards. Neither the crude nor the adjusted Cox multivariate analysis incontestable a statistically vital distinction within the hazard rate of HAI between exposure teams. The crude hazard rate of HAI for patients given tocilizumab was zero.97 times that of patients not given tocilizumab $(95 \% \mathrm{CI}=\mathrm{zero} .37,2.52, \mathrm{P}=0.943)$. The adjusted hazard rate of HAI for patients given tocilizumab was zero.85 times that of patients not given tocilizumab $(95 \% \mathrm{CI}=$ zero.29, 2.52, $\mathrm{P}=0.780$ ) when dominant for age, device days, BMI, diabetes, cancer, anti-inflammatory, and patient surgery.

\section{Discussion}

Tocilizumab is also a viable treatment for the CRS seen in later stages of COVID-19 infections. thanks to its mechanism of action and former information from the atrophic arthritis population, there's a priority concerning the potential redoubled risk of HAIs with tocilizumab administration. many clinical trials ar afoot that take a look at tocilizumab for the treatment of COVID-19 and our study will facilitate for developing treatment protocols. Our study was notable for the shortage of statistically completely different rates of HAIs and bacteriaemia, however a statistically vital distinction long of keep once comparison comparison patients UN agency did and failed to receive tocilizumab. we tend to hypothesized that patients UN agency received the IL- 6 substance would expertise the next incidence of HAI when dominant for confounders like age, device days, and alternative risk factors. Our finding of no redoubled secondary infection rates in COVID-19 patients treated with tocilizumab is soothing to clinicians UN agency have used or ar victimization this medical aid for the treatment of CRS. The CI of the adjusted hazard magnitude relation was between zero.29 and 2.52, however, creating the purpose estimate tough to interpret.

Other studies had similar findings with no vital variations in rates of bacteriaemia among those exposed to tocilizumab, though these were calculated as a secondary analysis. the most important experimental study of tocilizumab use in COVID-19 completed at Yale rumored a bacteriaemia rate of four-dimensional in patients administered tocilizumab, below rates rumored from China and ny town of V-E Day and 6 June 1944, respectively. Our rumored bacteriaemia rate was five.1\% within the patients UN agency were exposed to tocilizumab versus two.0\%(P=0.623). though slightly on top of that rumored at Yale, the distinction remains not statistically meaning. In our study, the sole statistically vital distinction between teams was the length of follow-up time. The patients exposed to tocilizumab had an extended time to event of infection, death, and/ or discharge. There was one ascertained outlier within the tocilizumab cluster that received tocilizumab on day twelve of hospitalization, that seemingly skew the results. However, the quality deviations were similar between each teams. Tocilizumab administration has been related to longer hospital stays, thought to ensue to each timings of drug administration and watching of potential complications. In our study, though matched for confounders, the patients UN agency received tocilizumab could have had associate degree ascertained higher clinical burden, which can conjointly need longer hospital stays. a extended hospital keep may even be explained by multiple factors like lack of clinical improvement, discomfort among suppliers, and a restricted provide of medication. This study was restricted by the study style as this wasn't a randomised management trial and is in danger of choice and indication bias. This was conjointly alittle sample size with a large CI which can be resulting in a kind II error. a real distinction in secondary infection rates could however exist, and a bigger study higher high-powered to discover such a distinction would supply additional info. one in every of the many limitations of this study was the challenge of process a secondary infection. The NHSN definition for secondary infections has strict diagnostic criteria that will take issue from clinical observe. Differentiating co-infection with microorganism or pneumonia on presentation, similarly as 
International Journal of Immunology \& Infectious Diseases ((IJIID) characteristic secondary pneumonias, was tough. though a poor potential marker of secondary pneumonias, ventilator-associated events were enclosed and sensitivity analyses that removed these from the analysis failed to modification the results ascertained. Finally, we tend to failed to follow patients on the far side discharge, that the that the of tocilizumab stay unknown.

\section{Conclusions}

Based on this study, it seems that receiving tocilizumab for the treatment of COVID-19 doesn't increase the incidence of secondary infections. Currently, there are a unit many randomised clinical trials that area unit afoot that check the protection and effectuality of tocilizumab for CRS in COVID-19, our trial will give some support concerning secondary infection rates.

\section{Research quality and ethics statement}

This study was approved by the Institutional Review Board / committee approval range 20-12-108-337(c20). The authors followed applicable EQUATOR Network (http://www.equator-network.org/) pointers throughout the conduct of this research.

\section{Financial support and support}

Nil.

\section{Conflicts of interest}

There aren't any conflicts of interest 


\section{References}

1.World Health Organization. Clinical Management of COVID-19: Interim Guidance 27 May 2020; c2020. Available from: https://apps.who.int/iris/ bitstream/handle/10665/332196/WHO-2019-nCoV-clinical-2020.5-eng. pdf?sequence=1\&isAllowed=y. [Last accessed on 2020 Oct 06].

2.Zhou F, Yu T, Du R, Fan G, Liu Y, Liu Z, et al. Clinical course and risk factors for mortality of adult inpatients with COVID-19 in Wuhan,China: A retrospective cohort study. The Lancet 2020;395:1054-1062.

3.Huang D, Lian X, Song F, Ma H, Lian Z, Liang Y, et al. Clinical features of severe patients infected with 2019 novel coronavirus: A systematic review and meta-analysis. Ann Transl Med 2020;8:576.

4.Copaescu A, Smibert O, Gibson A, Phillips EJ, Trubiano JA. The role of IL-6 and other mediators in the cytokine storm associated with SARSCoV-2 infection. J Allergy Clin Immunol 2020;146:518-340.

5.Ogata A, Kato Y, Higa S, Yoshizaki K. IL-6 inhibitor for the treatment of rheumatoid arthritis: A comprehensive review. Mod Rheumatol 2019;29:258-67.

6.Fu B, Xu X, Wei H. Why tocilizumab could be an effective treatment for severe COVID-19? J Transl Med 2020;18:164.

7.Keske Ş, Tekin S, Sait B, İrkören P, Kapmaz M, Çimen C, et al. Appropriate use of tocilizumab in COVID-19 infection. Int J Infect Dis 2020;99:338-43.

8.Liu B, Li M, Zhou Z, Guan X, Xiang Y. Can we use interleukin-6 (IL-6) blockade for coronavirus disease 2019 (COVID-19)induced cytokine release syndrome (CRS)? J Autoimmun 2020;111:102452.

9.Misra DP, Agarwal V, Gasparyan AY, Zimba O. Rheumatologists' perspective on coronavirus disease 19 (COVID-19) and potential therapeutic targets. Clin Rheumatol 2020;39:2055-62

Submit your next manuscript to Med Science Publishers and benefit from:

$\rightarrow$ Easy online submission process

$\rightarrow$ Rapid peer review process

$\rightarrow$ Online article availability soon after acceptance for Publication

$\rightarrow$ Open access: articles available free online

$\rightarrow$ More accessibility of the articles to the readers/researchers within the field

$\rightarrow$ Better discount on subsequent article submission

Submit your manuscript at

https://medsciencejournals.com/submit-manuscript 